\title{
EchoGéo
}

52 | 2020

Ho Chi Minh Ville, terrain de jeu(x) métropolitain(s)

\section{Ho Chi Minh Ville, terrain de jeu(x) métropolitain(s)}

Introduction

Marie Gibert-Flutre and Clément Musil

\section{(2) OpenEdition}

\section{Journals}

Electronic version

URL: https://journals.openedition.org/echogeo/19451

DOI: $10.4000 /$ echogeo.19451

ISSN: 1963-1197

\section{Publisher}

Pôle de recherche pour l'organisation et la diffusion de l'information géographique (CNRS UMR 8586)

\section{Electronic reference}

Marie Gibert-Flutre and Clément Musil, "Ho Chi Minh Ville, terrain de jeu(x) métropolitain(s)", EchoGéo [Online], 52 | 2020, Online since, connection on 10 August 2021. URL: http://journals.openedition.org/ echogeo/19451 ; DOI: https://doi.org/10.4000/echogeo.19451

This text was automatically generated on 10 August 2021.

EchoGéo est mis à disposition selon les termes de la licence Creative Commons Attribution - Pas d'Utilisation Commerciale - Pas de Modification 4.0 International (CC BY-NC-ND) 


\title{
Ho Chi Minh Ville, terrain de jeu(x) métropolitain(s)
}

\author{
Introduction
}

Marie Gibert-Flutre and Clément Musil

1 Dédier un dossier à une ville en particulier est une gageure mais, à l'heure où la métropolisation s'affirme comme un processus global majeur, les travaux situés et ancrés dans la spécificité de chacun des terrains urbains - avec leur chronologie et leurs trajectoires propres - nous semblent plus que jamais nécessaires pour en comprendre finement les inflexions et assemblages locaux (Roy et Ong, 2011). À ce titre, Ho Chi Minh Ville (ancienne Saigon), métropole du sud du Vietnam, avec ses 12 millions $d$ 'habitants, représente un laboratoire d'analyse particulièrement fertile pour repenser les mécanismes et les effets de ce processus protéiforme dans le contexte politique contemporain d'un État-Parti autoritaire, triplement héritier de relations étroites quoique toujours ambiguës - avec la Chine et sa diaspora, de la colonisation (1858-1954) et d'une période d'économie planifiée socialiste (1975-1986). Avec les réformes du Renouveau (Đổi Mới) initiées à partir de l'année 1986, le Vietnam a depuis adopté un modèle d'économie de marché à "orientation socialiste» qui s'est progressivement ouvert à l'économie mondialisée.

2 Le sous-titre du dossier «terrain de jeux métropolitain(s) » fait doublement référence aux jeux d'acteurs renouvelés auxquels donne lieu le processus de métropolisation contemporain de Ho Chi Minh Ville, mais aussi au retour en nombre des chercheurs étrangers sur le terrain vietnamien depuis la fin des années 1990, après des décennies marquées par la guerre et une relative fermeture du pays. Ho Chi Minh Ville permet ainsi de poser la question de la spécificité de la production métropolitaine, comme de la production scientifique, en contexte autoritaire. 


\section{Repenser les cadres interprétatifs de la métropolisation à partir d'une métropole dite « du Sud»}

3 Ce dossier dédié aux mutations contemporaines de Ho Chi Minh Ville s'inscrit pleinement dans le projet scientifique du Southern Turn, invitant à renouveler les cadres interprétatifs de la métropolisation à partir de terrains longtemps restés à la marge de la production conceptuelle en études urbaines (Connell, 2007; Edensor et Jayne, 2012 ; Parnell et Robinson, 2012 ; Perera et Tang, 2012, Sheppard et al., 2013; Parnell et oldfield, 2014). Ville asiatique colonisée - et de ce fait encore souvent associée à certains clichés - Ho Chi Minh Ville a d'abord été envisagée comme un simple terrain d'étude, et non comme un terrain de recherche permettant de repenser les cadres interprétatifs de la métropolisation et de forger des outils conceptuels renouvelés. Or, Nihal Perera et Wing-Shing Tang rappellent que «seeing and understanding [Asian] urbanisms, especially emerging spaces and transformative practices, are dramatically different exercises than collecting new data within existing intellectual and theoretical paradigm $»^{1}$ (Perera et Tang, 2013, p. 4). L'article de Charles Goldblum qui ouvre ce dossier explique ainsi que l'on ne peut envisager les métropoles vietnamiennes comme un simple récepteur de modèles mondialisés, car les logiques de ces derniers interfèrent immanquablement avec un jeu politique local complexe, fait de compromis et d'accommodements entre État et société (Duchère, 2019). Cet article précise alors comment le terrain urbain spécifique de Ho Chi Minh Ville permet en retour de penser une «fabrique du comparable» concernant l'analyse des formes de l'internationalisation urbaine en contexte asiatique.

4 La place grandissante des études vietnamiennes dans le champ de la géographie urbaine est aujourd'hui servie par le retour sur ce terrain de nombreux chercheurs, après des décennies marquées par les guerres et les contraintes géopolitiques. C'est également l'ambition de ce dossier de rendre compte de la production scientifique renouvelée qui caractérise Ho Chi Minh Ville aujourd'hui, où les travaux de longue durée, ancrés dans la complexité du terrain, se multiplient et couvrent des champs de plus en plus variés en études urbaines. Le bilinguisme de ce numéro, avec la participation de chercheurs français, mais aussi vietnamiens, canadiens et américains, atteste de la forte internationalisation des travaux sur une métropole où les conditions de production de recherche sont pourtant toujours régies par des contraintes fortes.

\section{Conduire des études métropolitaines en contexte autoritaire}

Ho Chi Minh Ville reste aujourd'hui un terrain de jeu métropolitain à l'accès contrôlé, requérant des méthodologies de recherche adaptées. L'accès aux données, notamment chiffrées, continue tout d'abord de poser des difficultés, tant les enjeux politicoéconomiques qui leur sont associés restent grands aux yeux de l'État-Parti qui les produit et les centralise. La question démographique en est le premier témoin: quantifier la population métropolitaine et sa croissance demeure un exercice de hautevoltige (Gubry, 2019). Le recensement officiel de la population exclut par exemple la population dite « flottante » des migrants ruraux, considérée comme temporaire, alors 
même qu'elle constitue aujourd'hui le moteur principal de la croissance démographique dans une métropole devenue un pôle d'attraction migratoire majeur à l'échelle nationale. Les étrangers sont également exclus des décomptes. Aux neuf millions d'habitants officiellement recensés dans la ville-province, s'ajouteraient en réalité plus de trois millions de personnes, pour un taux d'accroissement annuel de plus de $3 \%$ (Gubry, 2019).

6 Travailler sur l'urbain, c'est également toucher du doigt des opérations foncières et immobilières à forte plus-value, que l'on s'intéresse aux formes discrètes de la métropolisation dans les quartiers anciens de ruelles (Gibert-Flutre, 2019) ou aux projets urbains de grande envergure (Musil et Labbé, 2017 ; Musil et al., 2019). L'opacité demeure sur les modalités d'obtention des terrains et des permis de construire, sur la validation des projets d'aménagement et même sur l'interprétation des règles d'urbanisme, qui changent aussi vite que les formes de la ville elle-même (Quertamp, 2017). Les données associées à ces projets se caractérisent par ailleurs par leur caractère très fragmentaire et non suivi : il est très compliqué de tracer la généalogie des différents projets métropolitains et les jeux d'acteurs qui leur sont associés.

7 Au centre de toutes les tensions, les chercheurs butent immanquablement sur le nœud gordien des mutations métropolitaines de Ho Chi Minh Ville: le foncier, son statut juridique, sa valeur et ses modalités de transaction. Dans un pays où seul $30 \%$ de la population est titulaire d'un compte en banque, le foncier - en particulier en contexte métropolitain - fait office de placement et de produit d'investissement, au risque de contribuer à la fabrique de "quartiers fantômes » et de bulles spéculatives (Musil et al., 2019). Le foncier est aujourd'hui plus que jamais la clef de l'enrichissement à Ho Chi Minh Ville et documenter les logiques qui y président demeure une gageure pour les chercheurs, d'autant que certains acteurs centraux demeurent totalement inaccessibles, comme le ministère de la Défense, pourtant grand propriétaire foncier à Ho Chi Minh Ville. La récente destruction de l'ancienne zone portuaire coloniale de $\mathrm{Ba}$ Son, stratégiquement localisée dans le district péricentral de Bình Thạnh en bordure de la rivière Saigon, et la concession des terrains par ce ministère à de grands investisseurs privés pour y mener de vastes projets immobiliers de standing en est le signe le plus visible. Le complexe immobilier composé de 18 tours de grande hauteur du projet Vinhomes Central Park, dominé par l'emblématique Landmark 81 - tour la plus haute du Vietnam à ce jour-, symbolise parfaitement les mutations foncières, architecturales et sociales radicales de la période contemporaine. 
Illustration 1 - Une affiche officielle de l'État-Parti vietnamien devant les tours du projet immobilier Vinhomes Central Park

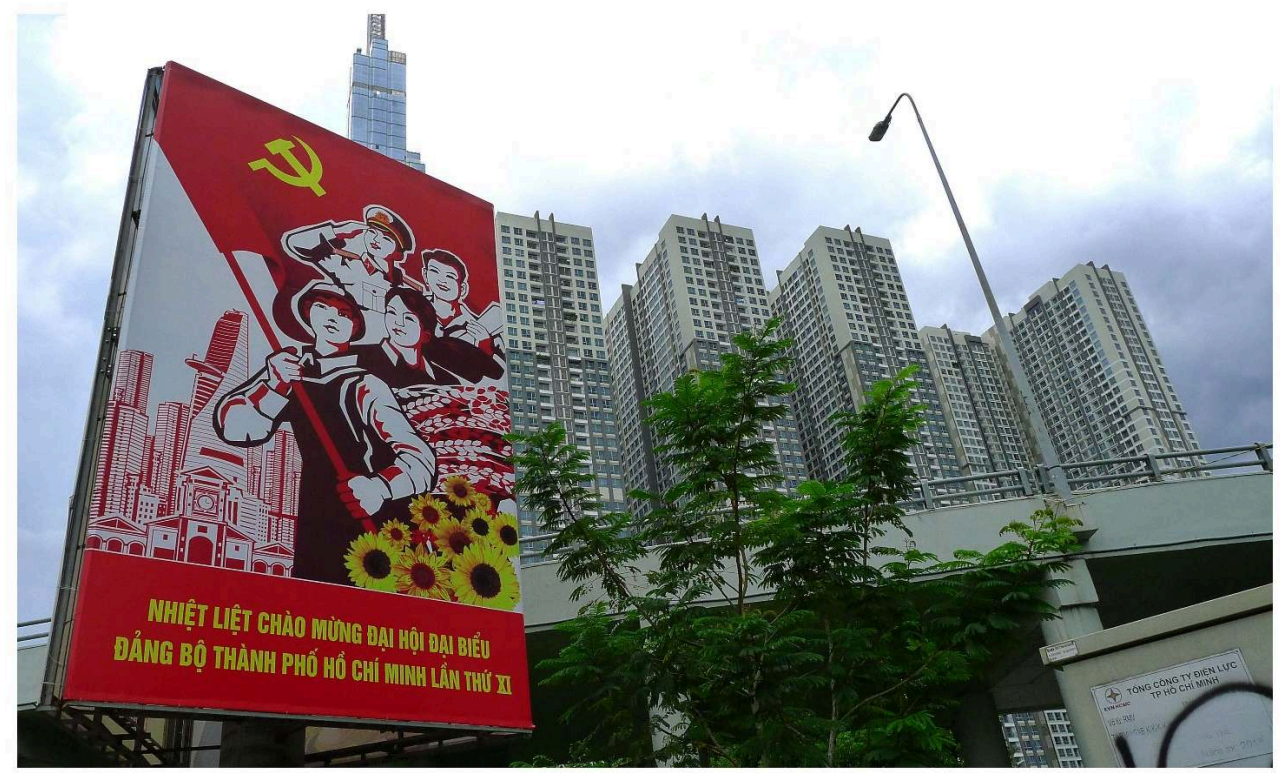

Traduction du slogan : « Salutations chaleureuses au $11^{\text {ème }}$ congrès du Parti communiste de Ho Chi Minh Ville».

Cette photographie datée de juillet 2020, quelques mois avant le XIème plenum du comité central du Parti communiste vietnamien (section de Ho Chi Minh Ville), met en regard les tours du projet Vinhomes Central Park (grand projet immobilier inauguré en 2018), symbole de la modernité métropolitaine de la ville-province, et une affiche de propagande d'État mettant en scène le Parti communiste - symbolisé par le marteau et la faucille en haut de l'affiche - et les forces majeures du développement du pays : l'ingénieur, le militaire, le col blanc et l'agriculteur. Cette affiche, aux couleurs officielles rouge et jaune, témoigne de la volonté de l'État-Parti vietnamien de contrôler les formes et modalités de globalisation du pays, et en particulier le processus de métropolisation de Ho Chi Minh Ville, incarné ici par le passage d'une ville horizontale à une ville verticale.

Auteur : C. Musil, 2020.

Il en va de même du réaménagement de la presqu'île de Thủ Thiêm dans le district 2. Ce projet titanesque au long cours incarne bien la complexité et les dérives des mutations métropolitaines de la ville, à l'heure du «virage immobilier» (Shatkin, 2017), ce que décrypte l'article de Clément Musil, Daniel Labbé et Vu Ngoc Khanh dans notre dossier. Alors que le marché immobilier de Ho Chi Minh Ville, notamment le segment résidentiel, est l'un des plus attractifs à l'échelle régionale de par la vitesse de son développement et ses rendements possibles (PwC et Urban Land Institute, 2020), les auteurs montrent combien le rôle des acteurs privés de l'immobilier est désormais prépondérant pour s'assurer de l'engagement des politiques publiques d'aménagement. Analyser les mutations métropolitaines et leurs ressorts fonciers, notamment le transfert de terrains dits publics vers des acteurs privés, dans un système où « la terre appartient au Peuple et l'État en assure la gestion ", c'est également croiser le chemin des commissions d'investigations de la lutte contre la corruption du Parti Communiste Vietnamien sans pouvoir accéder aux sources primaires nécessaires à l'analyse.

9 Ce contexte de recherche appelle en réponse des exercices de style complexes et invite à valoriser le travail inscrit dans le temps long de l'arpentage du terrain et de l'enquête qualitative, dont témoigne l'ensemble des articles de ce dossier. Rappelons par ailleurs que cette recherche en milieu autoritaire constitue avant tout une difficulté pour les chercheurs vietnamiens, qui font régulièrement face à des choix cornéliens mettant en jeu leur propre sécurité et la possibilité de poursuite de leur carrière dans la recherche 
publique. Dans ce contexte, les exercices de co-écriture d'articles entre chercheurs locaux et internationaux auxquels ont donné lieu plusieurs articles de ce dossier témoignent de l'importance d'établir de nouveaux partenariats de recherche internationaux pour mieux appréhender les subtilités d'un terrain qui se dérobe souvent à la vue et la compréhension immédiate.

\section{Ho Chi Minh Ville ou l'ambition métropolitaine d'un pays émergent}

Illustration 2 - La région métropolitaine de Ho Chi Minh Ville

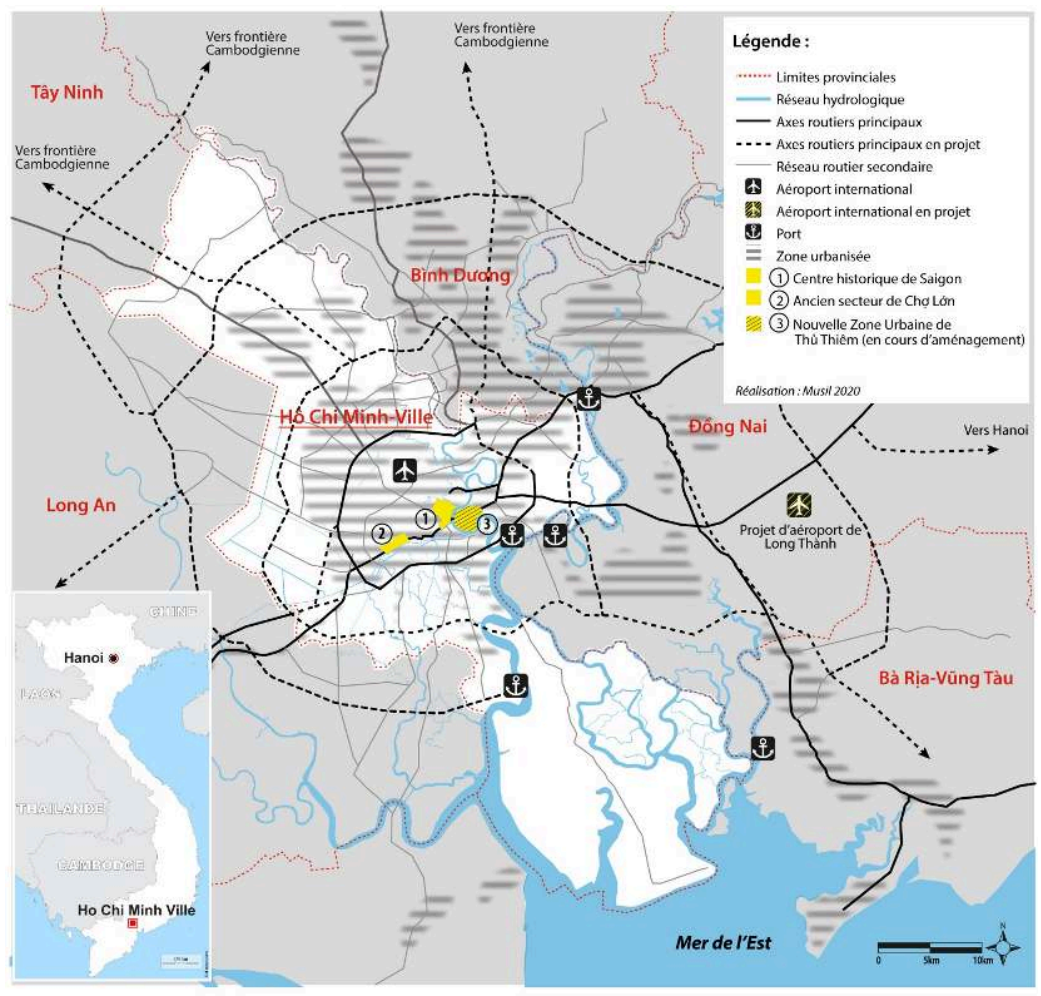

Auteur : C. Musil, 2020.

Comparativement aux plus de 1000 ans d'histoire de la ville de Hanoi, Saigon-Ho Chi Minh Ville est une ville qui demeure relativement "récente " dont la fondation officielle est communément datée de 1698. Cet ensemble urbain fut très tôt marqué par son ouverture sur l'étranger, incarnée en premier lieu par la présence historique des populations chinoises ayant participé à sa formation dans le quartier de chợ Lớn (Gibert-Flutre, 2017). L'histoire de la ville fut ensuite fortement infléchie par sa refondation coloniale entre 1859 et 1954, puis par son statut de sulfureuse capitale du Sud-Vietnam durant la guerre, avant que sa "libération » ou sa "chute ", selon la perspective idéologique retenue, ne l'inscrive dans la sphère des pays socialistes après 1975 (Drummond et Young, 2020).

De cette histoire mouvementée, Ho Chi Minh Ville garde des traces souvent invisibilisées aujourd'hui, comme autant de palimpsestes. La mise en valeur patrimoniale de cette histoire controversée n'est toujours pas à l'ordre du jour pour une ville que les autorités nationales cantonnent à un statut de métropole économique, 
par opposition à Hanoi située au cœur du berceau civilisationnel du Vietnam dans le delta du fleuve Rouge. L'histoire même de la ville peine encore à s'écrire et à trouver sa place dans le roman national. Cependant, son ouverture précoce à des influences étrangères diverses - visibles de manière discrète dans l'architecture, la gastronomie, la mode ou même certains modes de gestion administrative - explique qu'elle ait été le fer de lance de l'ouverture du pays dans les réseaux de la mondialisation dans les années post-réformes du Renouveau. La ville était alors la seule du pays à bénéficier d'infrastructures adéquates, mais surtout de précieuses inscriptions dans des réseaux d'investisseurs étrangers, notamment par le biais de la diaspora des Vietnamiens de l'étranger (Việt kiều).

\section{La grande mue métropolitaine}

12 Au tournant des années 1990, l'ouverture économique du Vietnam a été orchestrée par le Premier Ministre de l'époque, Võ Văn Kiệt, originaire du delta du Mékong, qui a agi en faveur d'une ouverture progressive - et sous contrôle - aux investissements directs étrangers (IDE). En raison du réseau d'infrastructures et du tissu économique hérités de la période de présence de l'armée américaine, et auparavant de la période de présence française, le décollage économique, désormais érigé en "miracle économique » (Vanham, 2018) a contribué à l'émergence d'un véritable «triangle de croissance » dès le début des années 1990 entre la ville-province de Ho Chi Minh Ville et les provinces voisines de Binh Duong, Dong Nai et Ba Ria Vung Tau (Luong, 2003 : 17-19). Cela s'est d'abord incarné par la création de zones industrielles reliées aux infrastructures portuaires routières "héritée " des périodes précédant la réunification du pays. En somme, la région métropolitaine de Ho Chi Minh Ville a servi de marchepied au décollage économique du pays, en attirant notamment des IDE. En 2019, cette région métropolitaine contribuait à plus de $40 \%$ du PIB national et à $60 \%$ du budget de l'État (Tractus, 2020). L'environnement réglementaire propice aux investissements, la présence d'infrastructures comme d'une main d'œuvre qualifiée a également permis à ce territoire d'attirer $60 \%$ des projets d'investissement et $50 \%$ des IDE à l'échelle nationale (ibid., 2020). Concevoir Ho Chi Minh Ville et sa région métropolitaine périphérique comme un moteur économique au service de l'intérêt du développement de la nation est toutefois trompeur. Comme le montre l'article de Tran Khach Minh, une forte compétition, voire des tensions, existe entre les provinces formant cette région industrielle pour attirer sur leur territoire les IDE comme les infrastructures de transport et les pôles logistiques. Ces rivalités entre territoires, tout comme l'ambition économique et industrielle de Ho Chi Minh Ville et sa région ne se limitent pas à ces chiffres. D'autres enjeux sont à venir.

13 La métropole se projette pleinement dans l'avenir de la production mondiale, en souhaitant tout d'abord bénéficier des délocalisations de sites de production initialement installés en Chine et dont le fonctionnement est aujourd'hui contrecarré par la guerre économique que se livrent les Etats-Unis et la Chine (Lam Thanh Ha et Nguyen Duc Phuc, 2019). L'ambition de Ho Chi Minh Ville est aussi de s'arrimer au mouvement global de l'industrie 4.0 (industrie à la convergence du monde virtuel - de la conception numérique, de la gestion des opérations, de la finance et du marketing avec les produits et objets du monde réel) en s'ancrant dans la « smart production ». Le Politburo du Parti Communiste vietnamien a déjà adopté une résolution dans ce sens (Kieu Phong et Thu Huong, 2019) et Vingroup, un conglomérat privé national qui a 
appuyé son développement sur des activités immobilières, se projette désormais sur le marché de l'intelligence artificielle, comme sur celui de la 5G (Jennings, 2020).

Reste que pour accroître ses performances économiques et industrielles, attirer de nouveaux IDE et de "nouveaux talents » indispensables aux start-up engagées dans l'industrie 4.0, la métropole est tenue de mettre à niveau ses infrastructures économiques de liaison. Une politique d'équipement volontariste a été validée par l'Etat-Parti au tournant des années 2000. Il s'agit d'assurer la construction de pôles logistiques (plateformes portuaires et aéroportuaires) pour faciliter l'import-export des produits assemblés ou confectionnés sur le territoire. En complément de l'aménagement de ports en eau profonde, situés à l'estuaire de la rivière Saigon, le gouvernement a récemment lancé un nouveau mégaprojet: la construction de l'aéroport métropolitain de Long Thành. La première phase de cet investissement doit s'achever fin 2025, en mobilisant un volume de près de 5 milliards de dollars US, alors que cette plateforme aéroportuaire va connaître des phases de développement jusqu'à l'horizon 2050, pour un investissement final de 16 milliards de dollars US (Hoang Phong, 2020). Il est aussi question de mettre à niveau les liaisons routières et autoroutières.

Le plan sectoriel des transports de Ho Chi Minh Ville, adopté en 2013, prévoit la réalisation de projets routiers (ponts, tunnels, parkings, rocades périphériques, routes en élévation, etc.) pour un volume d'investissement avoisinant les 40 milliards de dollars US d'ici à 2030 (Huu Cong, 2020). Ces investissements dans le secteur routier, indispensables selon les autorités pour asseoir le développement économique de la métropole, laissent entrevoir des changements dans les pratiques de déplacement et les mobilités du quotidien. C'est notamment la problématique abordée par l'article de HuêTâm Jamme qui, par son approche de géographie sociale, montre que les citadins, indépendamment de leurs catégories socio-professionnelles, attendent le passage de "la ville de la moto », caractéristique prégnante des villes vietnamiennes et de Ho Chi Minh Ville en particulier, à la "ville de l'automobile ", symbole d'une modernité (déjà dépassée ?). L'automobile constitue désormais un symbole fort de réussite économique pour les classes sociales moyennes et supérieures.

En dépit de cette tendance forte, le développement du transport public n'est pas en reste. Un vaste réseau de métro est planifié, avec plus de $200 \mathrm{~km}$ de linéaire et près de 8 lignes. Là encore, les investissements attendus sont colossaux : 18 milliards de dollars US d'ici à 2030 (Musil et Simon, 2015). Reste que, pour ces investissements, les ressources budgétaires sont limitées et le Vietnam est toujours dépendant d'une aide financière étrangère provenant des organisations internationales de développement. À la question du financement de ces équipements, s'additionnent d'autres problèmes : notamment la question récurrente des expropriations qui se posent immanquablement, aussi bien pour l'aéroport de Long Thành, pour une rocade périphérique que pour une ligne de métro, même souterraine (les accès aux stations, comme les dispositifs de ventilations et de sécurité incendie nécessitent l'accès au sol). Ces problèmes d'accès au foncier retardent les projets et font substantiellement augmenter leurs coûts dans la durée.

Le dernier aspect qui mérite d'être mentionné est le lancement de nouveaux types de projets de planification et d'urbanisation. Outre les questions de verticalisation, de densification, d'expansion et d'étalement urbain, qui posent des problèmes désormais majeurs pour l'adaptation des réseaux techniques et des services urbains (Musil et 
Labbé, 2018), la ville-province de Ho Chi Minh Ville planifie et souhaite réaliser un vaste projet connu sous le nom d'innovation district. Ce projet témoigne de la volonté de concrétiser le développement de Ho Chi Minh Ville comme une smart city. Mobilisant un grand nom de l'architecture mondialisée, la firme Sasaki, le projet prévoit de transformer 22000 ha répartis sur trois districts urbains à l'est de la métropole. Il s'agit en somme de traduire à travers un geste architectural et urbain l'entrée de Ho Chi Minh Ville dans l'industrie 4.0 et de doter la métropole de zones destinées à développer cette industrie, en s'appuyant sur les technologies nouvelles de l'information et de la communication afin de développer un Fin Tech Hub (pour une montée en puissance des services financiers) ou encore un IT \& Edu Tech Hub (pour la formation et la recherche et développement). En bref, il s'agit de planifier une série de nouveaux espaces à la fois tournés vers le développement des technologies tout en offrant une résilience face aux effets du changement climatique. De manière surprenante, ce projet a vocation à englober la nouvelle zone urbaine de Thủ Thiêm, dont le texte de l'anthropologue Erik Harms dans la rubrique "Sur l'Image », dresse le portrait avant les débuts de sa transformation, rappelant ainsi qu'elle n'était composée jusqu'à peu que d'espaces agricoles et de marécages, sur les bords de la rivière Saigon.

\section{La rivalité Hanoi/Ho Chi Minh Ville ou la stratégie du non-dit et de l'évitement}

Aborder l'ambition métropolitaine de Ho Chi Minh Ville, sa volonté de se placer comme un futur hub économique et financier à l'échelle régionale, c'est aussi poser la question du rapport entre la métropole du sud et la capitale du pays, Hanoi. S'il ne peut pas être affirmé qu'une rivalité existe entre ces deux villes, puisque le dessein national prime sur les concurrences entre territoires, des signaux faibles existent. Désormais $\mathrm{Ho} \mathrm{Chi}$ Minh Ville est affichée et estampillée comme la future smart city du Vietnam. Alors qu'Hanoi est déjà le siège du pouvoir politique, et présentée comme dépositaire de la culture vietnamienne, le gouvernement souhaite faire de la capitale une métropole régionale majeure en Asie. Pour cela, Hanoi se doit de dominer la hiérarchie urbaine vietnamienne et d'être identifiée comme la porte d'entrée du Vietnam (Logan, 2009). Récemment, le gouvernement a renouvelé son intention de favoriser la montée en puissance de la capitale en faisant de Hanoi un pôle majeur en Asie de l'Est et du SudEst d'ici 2045 (Việt Hùng et Văn Hưng, 2020).

Cette volonté n'est pas sans conséquence pour les autres centres urbains du pays. En clair, la priorité est de faire converger les investissements publics comme privés vers Hanoi, au détriment, supposément, des autres métropoles du pays. Or, Ho Chi Minh Ville peine déjà à trouver des financements pour réaliser les ambitieux projets exposés précédemment. Par exemple, la première ligne de métro de Ho Chi Minh Ville pour pouvoir être achevée et mise en service a besoin de rallonges budgétaires réclamées au gouvernement et qui tardent à venir. Or, la métropole du sud peut difficilement financer ses projets d'infrastructures par elle-même puisque le dispositif fiscal national impose une péréquation stricte entre les provinces; et Ho Chi Minh Ville ne conserve pas plus de $23 \%$ de ses recettes fiscales, notamment issues de l'activité économique générée localement (Nguyen Hoai, 2016). Sur fond de rivalités non dites entre Hanoi et la métropole du sud, apparait donc un déséquilibre grandissant entre l'ambition 
métropolitaine de Ho Chi Minh Ville et ses capacités réelles de financement de la mise aux normes de ses infrastructures.

Ces difficultés connaissent aujourd'hui un prolongement sur le terrain politique, voire judiciaire. En effet, depuis le lancement de la campagne anti-corruption du Parti Communiste Vietnamien en 2016, les cas de litiges identifiés par la commission d'investigation à Ho Chi Minh Ville se multiplient, mettant au jour des dysfonctionnements dans l'attribution de terrains publics (ou appartenant à des sociétés étatiques) à des promoteurs privés à Ho Chi Minh Ville (Le Hong Hiep, 2019). Plusieurs hauts fonctionnaires ont été depuis arrêtés, dont le plus notable est l'ancien membre du Politburo et secrétaire du Parti de Ho Chi Minh-Ville, Dinh La Thang. A travers cela, difficile de ne pas formuler l'hypothèse d'une volonté de maîtrise par Hanoi du développement de la métropole du sud. Cependant, en dépit de ces velléités politico-économiques, Ho Chi Minh Ville reste à ce jour la métropole économique la plus attractive du pays, à l'échelle nationale comme internationale.

\section{Et les citadins dans tout ça?}

21 Les citadins de Ho Chi Minh Ville occupent une place paradoxale dans cette grande mue métropolitaine et les jeux de pouvoir qui lui sont associés. Très largement exclus des processus officiels de prise de décisions, ils ne cessent néanmoins de s'inviter dans la fabrique de la ville par le recours à autant de tactiques, à la frontière poreuse entre formel et informel, légalité et illégalité (de Certeau, 1980 ; Gibert et Segard, 2015). La diversité des profils socio-économiques des habitants ne cesse par ailleurs de s'accroître, comme les inégalités qui les caractérisent.

La « classe moyenne ", dont il est particulièrement ardu de s'accorder sur des critères définitionnels simples dans le contexte vietnamien, est indéniablement au cœur des mutations métropolitaines. La ville peut se prévaloir d'une population toujours plus éduquée et qui ne cesse de s'enrichir : le PIB par habitant est trois à quatre fois plus élevé que la moyenne nationale et demeure supérieur à celui de Hanoi (Nguyễn Vũ, 2018 ; Tá Lâm, 2019). Cet enrichissement s'accompagne de nouvelles aspirations en termes de modes de vie et de consommation, auxquelles répondent les nouvelles offres du marché. Ces dernières concernent les logements - sous la forme de plus en plus répandue des condominiums, même si les petites maisons individuelles dominent toujours largement dans la ville -, la multiplication des shoppings malls et des espaces privés de sport et de loisirs ou encore les nouvelles offres de l'économie numérique de plateforme (avec la croissance très rapide de sites mondialisés de locations touristiques comme Airbnb et ses variantes nationales comme Luxstay, ou les nouvelles mobilités via la plateforme Grab par exemple). En raison d'une population de plus en plus connectée, le «e-commerce» fait figure de nouvelle pratique de consommation de masse, alors même que $10 \%$ seulement des paiements s'effectuent "en ligne " (Pimhidzai et al., 2019, p. 65). Malgré des changements rapides dans les modes de consommation, le recours à la transaction en espèces reste la norme. Les pratiques métropolitaines ne cessent de se diversifier, révélant parfois des tensions et adaptations entre modèles mondialisés et habitudes locales. La classe moyenne en expansion, par ailleurs très mise en valeur dans le marketing métropolitain, constitue un moteur essentiel de la croissance économique nationale, notamment pour «consommer " et absorber les productions manufacturières, comme immobilières. 
D'autres consommateurs apparaissent toutefois. Les consommateurs étrangers, notamment Sud-Coréens, Hong Kongais, ou d'Asie Sud-Est constituent désormais des cibles pour les promoteurs immobiliers locaux.

À cette classe moyenne et aux nouvelles élites économiques de la ville s'articulent les milieux populaires et une classe précaire, dont la diversité est également remarquable. La figure la plus visible de cette partie de la population est sans conteste celle des vendeurs de rue, dont les conditions d'exercice sont sujettes à autant d'arrangements locaux, avec la police et les riverains des quartiers où ils travaillent, à la pérennité très incertaine. Les migrants ruraux, nombreux à travailler comme ouvriers sur les chantiers ou dans les usines, constituent l'autre face visible de la précarité, condamnés à des salaires très faibles, des conditions d'exercice difficiles et des statuts administratifs tout aussi précaires. Les inégalités socio-spatiales ne cessent ainsi de se creuser et sont consubstantielles du processus de métropolisation de la ville, même si elles ne se traduisent pas toujours par une ségrégation spatiale. Certains quartiers notamment les nouvelles zones urbanisées de l'urbanisme de projet - sont très exclusifs par les prix fonciers et immobiliers affichés mais le tissu urbain global de la ville présente plutôt des interstices de pauvreté - notamment le long des canaux ou au cœur de quartiers de ruelles mal desservis - à proximité immédiate de quartiers de classe moyenne. Cette réalité se double de profondes articulations entre économie formelle et informelle, l'opposition de ces deux sphères n'ayant aucun sens dans le contexte vietnamien. Le secteur informel, caractérisé par les précarités des sources de revenus et la difficulté à les stabiliser dans le temps, ne cesse de se recomposer, notamment à l'heure de l'économie numérique de plateforme et l'on peut faire l'hypothèse de l'émergence d'une « informalité numérique » en cours d'émergence dans la métropole. Des vendeurs de rue ou des petites échoppes de quartiers précaires vendent ainsi leurs services en ligne et proposent des livraisons de repas ou de services. La grande mue métropolitaine s'accompagne donc de fortes recompositions des pratiques citadines aux effets souvent paradoxaux.

\section{A l'épreuve du siècle : quelles adaptations face aux effets du changement climatique?}

Comprendre les dynamiques métropolitaines contemporaines de Ho Chi Minh Ville c'est aussi souligner les singularités de ce territoire et sa géographie physique. Située au nord du delta du Mékong, à $70 \mathrm{~km}$ en amont de la mer de l'Est sur les bords de la rivière Saigon, Ho Chi Minh Ville présente un réseau hydrographique qui a été fortement anthropisé à partir du XVIII ${ }^{m e}$ s. (Vu Hong Lien, 2013) mais qui demeurait encore très faiblement urbanisé au moment de l'installation de l'armée française en 1859. Un officier de marine indiquait que «[...] au début de notre occupation, la partie basse de Saigon n'était guère qu'une plaine marécageuse couverte par les eaux à chaque grande marée » (extrait cité in Bouchot, 1927, p. 73).

L'urbanisation de l'ancienne Saigon débuta par des travaux de voirie et l'édification de bâtiments emblématiques (dont certains font désormais figure de patrimoine architectural, comme la poste ou encore la cathédrale), mais surtout par des travaux d'aménagement hydrauliques majeurs comprenant le comblement de canaux et le percement de nouveaux canaux (Gibert-Flutre, 2019, p.63-70). La région de $\mathrm{Ho}$ Chi Minh Ville n'a cependant pas connu de vastes travaux d'endiguement des fleuves et 
rivières, à la différence de Hanoi et de la région du delta du Fleuve Rouge dont le territoire a été façonné par des siècles de construction de digues (Ha Phong Le, 2016) et d'aménagement de casiers agricoles dédiés à la riziculture (Fanchette et al., 2015). Au début du XXI ${ }^{\mathrm{eme}}$ s., dans le contexte global de changement climatique, l'omniprésence de l'eau constitue une particularité prégnante et identitaire de Ho Chi Minh Ville, mais également un risque.

Depuis plusieurs années, les travaux du Groupe d'experts intergouvernemental sur l'évolution du climat (Giec) indiquent que les effets du changement climatique augmentent le pouvoir destructeur des inondations liées aux pluies extrêmes et à la montée des eaux de mers et de rivières (IPCC, 2018). Au milieu des années 2000, Ho Chi Minh Ville fut identifiée comme l'une des 10 métropoles au monde les plus vulnérables aux effets du changements climatiques (Stern et al., 2006; Hanson et al., 2011). Cette vulnérabilité est d'autant plus importante que le risque d'inondation extrême pourrait augmenter de 5 à 10 fois d'ici 2050 (Woetzel et al., 2020). En outre, près de $60 \%$ de la surface bâtie est située sur des sols dont l'altitude s'élève à moins de 2 mètres par rapport au niveau de la mer. À la fin des années 2000, c'était déjà environ $26 \%$ de la population de HCMV qui était affectée par les inondations lors de tempêtes violentes, soit environ 2,5 millions d'habitants (ADB, 2010, p. 11). Les travaux de prospective dans ce domaine sont loin d'être optimistes: d'ici à 2050 ces événements climatiques toucheraient entre 6 et 9 millions d'habitants selon les mesures de protections et d'aménagement qui pourraient être mis en place d'ici là ((Woetzel et al., 2010, p. 11). Dans leur contribution à ce numéro, Georges Vachaud, Nicolas Gratiot et Tien Dung Tranh Ngoc reviennent sur les enjeux multiples liés aux inondations et prolongent leur propos en discutant de manière critique des stratégies de gestion des risques envisageables et adaptées au contexte de Ho Chi Minh Ville.

Le développement urbain actuel marqué par la construction de nouvelles zones urbaines, de zones industrielles et d'infrastructures de transport, ne cesse d'accroître la vulnérabilité de la métropole. D'un côté la métropole est confrontée à un phénomène de subsidence (Dinh et al., 2015) et de l'autre à une accélération de l'artificialisation des sols qui amenuise les possibilités d'évacuation des eaux lors des crues des rivières et canaux, au moment des pluies abondantes et des forts coefficients de marée. Entre 1990 et 2012 , ce sont près de 3000 hectares de terres qui ont été annuellement urbanisés (Kontgis et al., 2014). En plus des coûts humains, les événements climatiques les plus violents risquent d'endommager les réseaux d'infrastructures (transport, communication, énergie, eau). Pour 2050, l'un des scenarii évoqués suggère l'endommagement (voir la destruction) de plus de 8 milliards de dollars US d'actifs immobiliers, d'environ 1 milliard de dollars US d'infrastructures (dont ports et usines de traitement des eaux), et de près de 8 milliards de dégâts relatifs aux services urbains (immobilisation du futur réseau de métro). Il existe des scenarii encore plus alarmants si l'élévation du niveau des mers dépasse les $180 \mathrm{~cm}$ à compter de 2050. En somme, c'est l'urbanisation en cours, à savoir les projets immobiliers et industriels comme les infrastructures de transport très coûteuses, qui sont, avant même d'être achevées, déjà considérés comme exposés et vulnérables aux effets du changement climatique (Woetzel et al., 2020).

28 Mais l'ambition métropolitaine, couplée à l'optimisme tant des autorités publiques et politiques que de la population, rendent envisageable l'adoption et la mise en œuvre de plan de protection, et potentiellement leur réussite. Avec l'appui de partenaires 
étrangers, notamment avec la Banque Asiatique de Développement d'un côté et la ville de Rotterdam de l'autre, les services techniques de Ho Chi Minh Ville ont conduit des études et ont amené les dirigeants politiques à adopter en 2013 un plan d'action visant l'adaptation au changement climatique et l'atténuation de ses effets. Ce plan prévoit en particulier la conduite d'études complémentaires, mais surtout des projets techniques d'endiguement des secteurs les plus exposés de la métropole, ou encore la construction de parcs urbains faisant office de futurs bassins de rétentions. La volonté politique se traduit enfin par des adaptations institutionnelles - voire des innovations -, dans le champ de la gouvernance urbaine poussant à repenser la déconcentration institutionnelle qui marque le fonctionnement de l'administration territoriale.

La menace environnementale constitue sans conteste le défi du siècle pour les ambitions métropolitaines de Ho Chi Minh Ville et pèse déjà sur ses perspectives de croissance économique à moyen terme. La capacité de réponse à cette menace multiforme fait alors figure de test pour l'État-Parti, dans un pays où l'harmonie et la stabilité sont érigées en valeurs cardinales. La récente lutte contre la Covid-19, évoquée dans le texte de Christophe Robert dans la rubrique Sur l'Image, a esquissé des pistes de réponse, entre réactivation des modalités de contrôle sanitaire et social héritées de l'époque socialiste et leur articulation nouvelle avec l'usage des nouvelles technologies. Mais si, à l'heure de boucler ce dossier, le succès semble au rendez-vous dans l'endiguement de la pandémie dans le pays, les crises économiques et sociales qui lui sont corrélées constituent autant d'incertitudes dans les jeux d'acteurs métropolitains à venir.

\section{BIBLIOGRAPHY}

Asian Development Bank, 2010. Ho Chi Minh City Adaptation to Climate Change: Summary Report. Mandaluyong City, Philippines, Asian Development Bank, 37 p. URL: https://www.adb.org/sites/ default/files/publication/27505/hcmc-climate-change-summary.pdf

Bouchot J., 1927. La naissance et les premières années de Saigon ville française. Saigon, Editions Albert Portail, $80 \mathrm{p}$.

Certeau, de M., Giard L. et Mayol P., 1980. L'invention du quotidien. 1 Arts de Faire. Paris, Galimard, $349 \mathrm{p}$.

Connell R., 2007. Southern Theory: The Global Dynamics of Knowledge in Social Science. s.l., Allen \& Unwin, $288 \mathrm{p}$.

Dinh H.T.M., Le Van T., Le Toan T., 2015. « Mapping Ground Subsidence Phenomena in Ho Chi Minh City through the Radar Interferometry Technique Using ALOS PALSAR Data ». Remote Sensing, vol. 7, $\mathrm{n}^{\circ}$ 7, p. 8543-8562.

Drummond L.B. et Young D. (ed.), 2020, Socialist and Post-Socialist Urbanisms: Critical Reflections from a Global Perspective. Toronto, University of Toronto Press, $336 \mathrm{p}$. 
Duchère Y., 2019, Hà Noi et sa région. Une géographie du compromis en régime autoritaire. Paris, Les Indes savantes, $238 \mathrm{p}$.

Edensor T. et Jayne M. (ed.), 2012, Urban Theory beyond the West: a World of Cities. London - New York, Routledge, $382 \mathrm{p}$.

Fanchette S., Orfeuvre R., Trần N.K., 2015, « Hà Nội entre les "eaux" ». In Fanchette S. (ed.), Hà Nội, future métropole. Rupture dans l'intégration urbaine des villages. Marseille, IRD Éditions, p. 19-38.

Gibert M., Segard J., 2015. « L'aménagement urbain au Vietnam, vecteur d'un autoritarisme négocié ». Justice spatiale, Spatial justice [En ligne], n 8. URL: http://www.jssj.org/article/ lamenagement-urbain-au-vietnam-vecteur-dun-autoritarisme-negocie/

Gibert-Flutre M., 2019. Les envers de la métropolisation. Les ruelles de Hồ Chí Minh Ville (Vietnam). Paris, CNRS Éditions, 243 p.

Gibert-Flutre Marie, 2017, « Chợ Lớn, la trajectoire paradoxale d'un chinatown historique ». In Sanjuan T. (ed.), Les chinatowns. Trajectoires urbaines de l'identité chinoise à l'heure de la mondialisation. Paris, UMR Prodig, p. 43-75.

Gubry P., 2019. « Résultats préliminaires du recensement du Viêt-nam du 1er avril 2019 : une réflexion sur l'effectif de la population de Hanoi et Hô Chi Minh Ville ». La lettre de l'AFRASE, n 96, p. 58-65.

Ha P. Le, 2016, Les enjeux territoriaux contemporains de l'endiguement en milieu urbain. L'évolution des rives du fleuve Rouge à Hanoï. Thèse en Architecture et aménagement de l'espace, Université de Lyon, Lyon, 615 p.

Hanson S., Nicholls R., Ranger N. et al., 2011. « A Global Ranking of Port Cities with High Exposure to Climate Extremes ». Climatic Change, vol. 104, n 1, p. 89-111.

Hoang P., 2020. « Work on Long Thanh Airport can begin in May 2021: ACV ». VN Express International, 16 avril.

Huu C., 2020. « HCMC traffic projects require $\$ 39$ billion over next decade ». VN Express International, 2 juillet.

IPCC (Intergovernmental Panel on Climate Change), 2018. « Summary for Policymakers ». In Global Warming of $1.5^{\circ} \mathrm{C}$. An IPCC Special Report on the impacts of global warming of $1.5^{\circ} \mathrm{C}$ above preindustrial levels and related global greenhouse gas emission pathways, in the context of strengthening the global response to the threat of climate change, sustainable development, and efforts to eradicate poverty, IPCC, p. 24. URL: https://www.ipcc.ch/sr15/chapter/spm/

Jennings R., 2020. « Vietnamese Billionaire's Conglomerate Develops Landmark 5G Smartphone ». Forbes, 6 juillet.

Kieu P., Thu H., 2019. « HCMC leading Vietnam in Industry 4.0 ». Sài Gòn Giải Phóng, 18 novembre. URL: https://sggpnews.org.vn/hochiminhcity/hcmc-leading-vietnam-in-industry-40-84448.html

Kontgis C., Schneider A., Fox J., Saksena S. et al., 2014, « Monitoring Peri-Urbanization in the Greater Ho Chi Minh City Metropolitan Area ». Applied Geography [En ligne], vol. 53, p. 377-388. DOI: https://doi.org/10.1016/j.apgeog.2014.06.029

Lam T.H., Nguyen D.P., 2019. « The US-China Trade War: Impact on Vietnam ». ISEAS Perspective, $\mathrm{n}^{\circ}$ 102, 13 p. URL: https://www.iseas.edu.sg/wp-content/uploads/pdfs/

ISEAS_Perspective_2019_102.pdf 
Le H. H., 2019. « The Impact of Vietnam's Anti-corruption Campaign on the Real Estate Sector ». ISEAS Perspective, $\mathrm{n}^{\circ}$ 46, 7 p. URL: https://www.iseas.edu.sg/images/pdf/

ISEAS_Perspective_2019_46.pdf

Logan W.S., 2009. « Hanoi, Vietnam ». City: analysis of urban trends, culture, theory, policy, action, vol. 13, nº 1, p. 87-94. DOI : https://doi.org/10.1080/13604810902726251

Luong H. V. (ed.), 2003. Postwar Vietnam: Dynamics of a Transforming Society. Singapore, ISEAS Publications, $336 \mathrm{p}$.

Musil C., Labbé D., 2018. « Frénésie immobilière au Vietnam Expansion ou étalement et surdensification? ». Études Foncières, $n^{\circ} 26$, p. 25-30.

Musil C., Labbé D., 2017. « Projets et négociations au Vietnam. Les moteurs de la conversion foncière urbaine ». In Peyronnie K., Goldblum C., Sisoulath B. (ed.), Transitions urbaines en Asie du Sud-Est. De la métropole émergente et de ses formes dérivées. Marseille, IRD Éditions, IRASEC, p. 191-2016.

Musil C., Labbé D., Jacques O., 2019, « Les zones urbaines "fantômes” de Hanoi (Vietnam). Éclairage sur le (dys)fonctionnement d'un marché immobilier émergent ». Cybergeo: European Journal of Geography [En ligne], n882. DOI : https://doi.org/10.4000/cybergeo.31466

Musil C., Simon C., 2015. « Building an Ambitious Public Transport System in Ho Chi Minh City (Vietnam)». Working Papers Series, Urban Development Management Support Center (PADDI), $\mathrm{n}^{\circ} 1$, 13 p. URL: https://www.codatu.org/wp-content/uploads/WP_PADDI_01_ENG_low-quality1.pdf

Nguyen H., 2016. « HCMC warns proposed budget cuts could have "dire consequences" ». VN Express International, 23 octobre. URL: https://e.vnexpress.net/news/business/hcmc-warnsproposed-budget-cuts-could-have-dire-consequences-3487942.html

Nguyễn V., 2018. « Năm 2018, kinh tế-xã hội Thủ đô đạt kết quả toàn diện [En 2018, le secteur économique et social de la capitale a atteint les résultats escomptés] ». Portail d'informations électroniques du département de la planification et de l'investissement de la ville de Hanoi, 2 décembre. URL: http://hapi.gov.vn/vi-VN/nam-2018-kinh-te-xa-hoi-thu-do-dat-ket-qua-toan-dienc59t1n12228

Parnell S., Oldfield S. (ed.), 2014. The Routledge Handbook on Cities of the Global South. New York, Routledge, $635 \mathrm{p}$.

Parnell S., Robinson J., 2012. « (Re)theorizing Cities from the Global South: Looking Beyond Neoliberalism ». Urban Geography [En ligne], vol. 33, nº 4, p. 593-617. DOI: https://doi.org/ 10.2747/0272-3638.33.4.593

Perera N., Tang W.-S. (ed.), 2012. Transforming Asian Cities: Intellectual Impasse, Asianizing Space, and Emerging Translocalities. London-New York, Routledge, 320 p.

Pimhidzai O., Lebrand M.S.M., Skorzus R.C., et al., 2019. Vietnam Development Report 2019: Connecting Vietnam for Growth and Shared Prosperity. Washington, D.C., World Bank Group, 150 p. URL: https:// documents.worldbank.org/en/publication/documents-reports/documentdetail/ 590451578409008253/vietnam-development-report-2019-connecting-vietnam-for-growth-andshared-prosperity

PwC et Urban Land Institute, 2019. Emerging Trends in Real Estate. Asia Pacific 2020. Washington, D.C, PwC and the Urban Land Institute, 54 p. URL: https://www.pwc.com/sg/en/publications/assets/ emerging-trends-real-estate-asia-pacific-2020.pdf 
Quertamp F., 2017. « Hô Chi Minh-Ville. Une planification centralisée à l'épreuve des grands projets ». In Peyronnie K., Goldblum C., Sisoulath B. (ed.), Transitions urbaines en Asie du Sud-Est. De la métropolisation émergente et de ses formes dérivées. Marseille, IRD Éditions, IRASEC, p. 81-108.

Roy A., Ong A. (ed.), 2011. Worlding cities: Asian Experiments and the Art of Being Global. MaldenOxford-Chichester, Wiley-Blackwell, $352 \mathrm{p}$.

Shatkin G., 2017. Cities for Profit: The Real Estate Turn in Asia's Urban Politics. Ithaca, Cornell University Press, 296 p.

Sheppard E., Leitner H. Maringanti A., 2013. « Provincializing Global Urbanism: A Manifesto ». Urban Geography [En ligne], vol. 34, nº 7, p. 893-900. DOI: https://doi.org/ 10.1080/02723638.2013.807977

Stern N., Peters S., Bakhshi V., 2006. The Economics of Climate Change: The Stern Review. Cambridge, Cambridge University Press, vol. 6, URL: http://www.lse.ac.uk/granthaminstitute/publication/ the-economics-of-climate-change-the-stern-review/

Tá L., 2019, « TP.HCM khó đạt thu nhập 9.800 USD/người vào năm 2020 [Le PIB de Ho Chi Minh Ville atteindra 9800 USD d'ici 2020] ». Báo Pháp Luật TP. Hồ Chí Minh, 31 mars. URL: https://plo.vn/ thoi-su/tphom-kho-dat-thu-nhap-9800-usd-nguoi-vao-nam-2020-824796.html

Tractus, 2020. « Vietnam Southern Economic Region ». Tractus website, 7 p. URL: http://tractusasia.com/wp-content/uploads/2019/03/Vietnam-Southern-Key-Economic-Regions-2018.pdf

Vanham P., 2018. « The Story of Viet Nam's Economic Miracle ». World Economic Forum on ASEAN, 11 septembre. URL: https://www.weforum.org/agenda/2018/09/how-vietnam-became-aneconomic-miracle/

Việt H., Văn H., 2020. « Thủ tướng: Đến 2045, Hà Nội phải đạt tầm nhìn là trung tâm Đông Á [D'après le Premier ministre : d'ici 2045, Hanoi doit poursuivre l'ambition de devenir le centre de l'Asie orientale] ». Zing News, 27 juin. URL: https://zingnews.vn/thu-tuong-den-2045-ha-noi-phaidat-tam-nhin-la-trung-tam-dong-a-post1100638.html

Vu H.L., 2013. The Making of Saigon from the Nguyen Lords to 1954. London, British Academy and ECAF, $36 \mathrm{p}$.

Woetzel J., Pinner D., Samandari H. et al., 2020. Can Coastal Cities Turn the Tide on Rising Flood Risk? McKinsey Global Institute, 29 p. URL: https://www.mckinsey.com/ /media/McKinsey/ Business\%20Functions/Sustainability/Our\%20Insights/ Can\%20coastal\%20cities\%20turn\%20the\%20tide\%20on\%20rising\%20flood\%20risk/MGI-Cancoastal-cities-turn-the-tide-on-rising-flood-risk.pdf

\section{NOTES}

1. «Prendre en compte et décrypter les urbanismes d'Asie, en particulier les espaces émergents et les pratiques génératrices de mutations, sont des démarches tout à fait différentes de celles qui consistent à collecter de nouvelles données dans un cadre interprétatif théorique existant ». 


\section{AUTHORS}

\section{MARIE GIBERT-FLUTRE}

Marie Gibert-Flutre, marie.gibert@univ-paris-diderot.fr, est maître de conférences en géographie à l'Université Paris de Paris (UFR LCAO) et membre de l'UMR CESSMA. Elle a récemment publié : - Gibert-Flutre Marie, 2020, L'Asie du Sud-Est, Paris, CNRS Éditions, 64 p.

- Gibert-Flutre Marie, 2019, Les envers de la métropolisation. Les ruelles de Hồ Chí Minh Ville (Vietnam), Paris, CNRS Éditions, 243 p.

- Gibert-Flutre Marie, 2018, « Rethinking Metropolitan Production from its Underside: A View from the Alleyways of Hồ Chí Minh City ", Environment and Planning A: Economy and Space, 2018, vol. 50, $\mathrm{n}^{\circ} 3$, p. 589-607.

\section{CLÉMENT MUSIL}

Clément Musil, musil.clement@gmail.com, est chercheur associé à l'UMR AUSser 3329 et à la chaire de recherche à l'Université de Montréal (Canada). Il a notamment publié :

- Musil C., 2020. Hong Kong's “Rail-plus-Property” development, a model for financing public transport in South-East Asian developing cities?. In Rengarajam P., Bing Z., Rabé P. (ed.), Future Challenges of Cities. IIAS: Leiden (Netherlands), Amsterdam University Press.

_ Musil C., Labbé D., Jacques, O., 2019. Les zones urbaines « fantômes » de Hanoi (Vietnam). Éclairage sur le (dys)fonctionnement d'un marché immobilier émergent. Cybergéo: European Journal of Geography [En ligne]. URL: https://journals.openedition.org/cybergeo/31466 - DOI: https://doi.org/10.4000/cybergeo.31466

- Nguyen T. B., Van Der Krabben E., Musil C., Le A.D., 2018. "Land for Infrastructure" in Ho Chi Minh City: Land-based Financing of Transportation Improvement. International Planning Studies [En ligne], vol. 23, n 3, p. 310-326. URL: https://www.tandfonline.com/doi/abs/ 10.1080/13563475.2018.1477581 - DOI: https://doi.org/10.1080/13563475.2018.1477581 